\title{
Observation of high Rydberg states of one-dimensional excitons in GaAs quantum wires by magnetophotoluminescence excitation spectroscopy
}

\section{$\operatorname{AUTHOR}(\mathrm{S}):$}

Okano, Makoto; Kanemitsu, Yoshihiko; Chen, Shaoqiang; Mochizuki, Toshimitsu; Yoshita, Masahiro; Akiyama, Hidefumi; Pfeiffer, Loren N.; West, Ken W.

\section{CITATION:}

Okano, Makoto ... [et al]. Observation of high Rydberg states of one-dimensional excitons in GaAs quantum wires by magneto photoluminescence excitation spectroscopy. Physical Review B 2012, 86(8): 085312.

\section{ISSUE DATE:}

\section{2-08-16}

\section{URL:}

http://hdl.handle.net/2433/159454

RIGHT:

(C)2012 American Physical Society 


\title{
Observation of high Rydberg states of one-dimensional excitons in GaAs quantum wires by magnetophotoluminescence excitation spectroscopy
}

\author{
Makoto Okano, ${ }^{1, *}$ Yoshihiko Kanemitsu, ${ }^{1}$ Shaoqiang Chen, ${ }^{2}$ Toshimitsu Mochizuki, ${ }^{2}$ Masahiro Yoshita, ${ }^{2}$ \\ Hidefumi Akiyama, ${ }^{2}$ Loren N. Pfeiffer, ${ }^{3}$ and Ken W. West ${ }^{3}$ \\ ${ }^{1}$ Institute for Chemical Research, Kyoto University, Uji, Kyoto 611-0011, Japan \\ ${ }^{2}$ Institute for Solid State Physics, University of Tokyo, Kashiwa, Chiba 277-8581, Japan \\ ${ }^{3}$ Department of Electrical Engineering, Princeton University, Princeton, New Jersey 08540, USA \\ (Received 5 March 2012; revised manuscript received 29 July 2012; published 16 August 2012)
}

\begin{abstract}
We have studied the higher Rydberg states of the ground exciton in $T$-shaped GaAs quantum wires with low-temperature photoluminescence excitation spectroscopy under magnetic fields. The peak of the second even-parity Rydberg state appears and grows in intensity at the onset of the one-dimensional (1D) continuum edge under strong magnetic fields. In stark contrast to the ground exciton, it shows significantly low zero-field oscillator strength and a large diamagnetic shift. These characteristic features are attributed to the extremely weakly bound wave function of the second Rydberg state, reflecting the inherent and novel character of 1D excitons that was predicted theoretically.
\end{abstract}

DOI: 10.1103/PhysRevB.86.085312

PACS number(s): 78.67.Lt, 71.35.-y, 78.20.Ls, 78.55.Cr

\section{INTRODUCTION}

The optical properties of one-dimensional (1D) quantum structures have attracted considerable interest from the viewpoint of fundamental physics and for high-performance device applications. ${ }^{1}$ Because of the spatial carrier confinement in 1D structures, the strong Coulomb interaction between conduction-band electrons and valence-band holes causes stable excitons to be formed. ${ }^{2}$ Optical transitions are concentrated at the lowest exciton, and the band-to-band absorption is either extremely weak or not observed in the linear absorption spectra. ${ }^{3,4}$ Because the stable exciton plays an important role in determining optical properties, the excitonic structures in various 1D nanomaterials, such as GaAs quantum wires, ${ }^{5,6}$ carbon nanotubes ${ }^{7-9}$ and Si polymers,${ }^{10}$ have been extensively studied. Recently, remarkable progress has been achieved in high-quality 1D quantum-structure fabrication methods, ${ }^{11-14}$ indicating that a clear and direct observation of excited-exciton states in 1D systems is possible. In particular, 1D GaAs wires fabricated by molecular-beam epitaxy (MBE) offer some advantages for use in optoelectronic devices because of their embedded structures. ${ }^{15}$

Numerous studies have been conducted on the excitedexciton states in semiconductor quantum wires. ${ }^{6,16-24}$ The observed excited-exciton states consist of higher electron and higher hole subbands or different electron and hole subbands. The higher Rydberg states of the ground exciton, consisting of the first electron and the first hole subbands, are indistinguishable from the band edge and other possible excitedsubband transitions because of their small binding energies and broadening. Thus, the higher Rydberg state excitons in GaAs quantum wires with a quantum number $n>1$ have never been verified to date. However, the experimental verification of the higher Rydberg state excitons is very important.

Theoretically, peculiar characteristics of $1 \mathrm{D}$ exciton structures are predicted to manifest themselves in the form of the stark contrast between the properties of the lowest and higher Rydberg states of 1D excitons. ${ }^{3,4}$ Both the binding energy and the oscillator strength of the lowest Rydberg states are significantly increased as $1 \mathrm{D}$ confinement becomes stronger, whereas those of the higher Rydberg states are expected to typically decrease ${ }^{3}$ and may show anomalous dependence of 1D excitons on the effective 1D Coulomb interaction strength. ${ }^{4}$ Despite their significance in 1D exciton physics, the detailed properties of higher Rydberg state excitons have not been determined experimentally thus far for any of the $1 \mathrm{D}$ nanostructures, including carbon nanotubes. In order to gain an insight into the nature of $1 \mathrm{D}$ excitons, a direct comparison between the lowest and higher Rydberg states of 1D excitons is needed. Moreover, a better understanding of high-energy exciton states would provide a detailed insight into nonlinear optical processes and multiexciton processes such as quantized Auger recombination ${ }^{25-27}$ and multiple exciton generation. ${ }^{28,29}$

In this article, we report the first direct observation of the higher Rydberg states of 1D excitons using multidirectional magneto-photoluminescence excitation (PLE) spectroscopy at low temperatures, which provides systematic and quantitative access to higher Rydberg state excitons in 1D systems. PLE spectra of $T$ wires show anisotropic magnetic-field dependence, providing direct experimental evidence of $T$-shaped quantum confinements. These magnetic effects intensify the peaks in the PLE spectra of $T$-wire excitons and reveal the internal structure of 1D excitons. The second even-parity Rydberg state appeared near the 1D continuum edge, and its intensity increased with the magnetic field. We observed low oscillator strength and a large diamagnetic blueshift for the second Rydberg state, in contrast to those of the exciton ground state. These observations reveal an intriguing contrast between the strongly and weakly bound wave functions of the ground and the higher Rydberg states of $1 \mathrm{D}$ excitons. This contrast can be attributed to the inherent characteristics of 1D excitons.

\section{EXPERIMENT}

Highly uniform 100-period $T$ wires were fabricated by MBE with cleaved-edge-overgrowth and growth-interrupt annealing techniques. ${ }^{11,12}$ Figure 1(a) shows the cross-sectional scanning transmission electron microscopy (TEM) image of a $100 T$-wire laser structure. The $100 T$ wires were formed at 


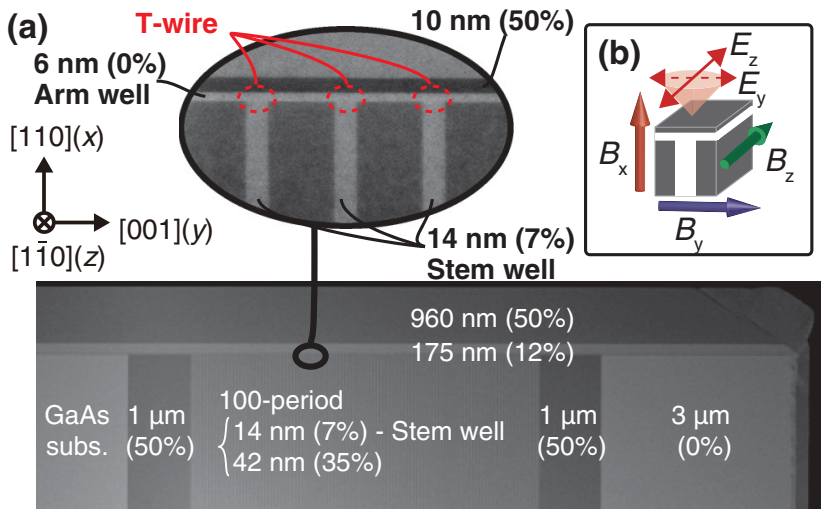

(c)

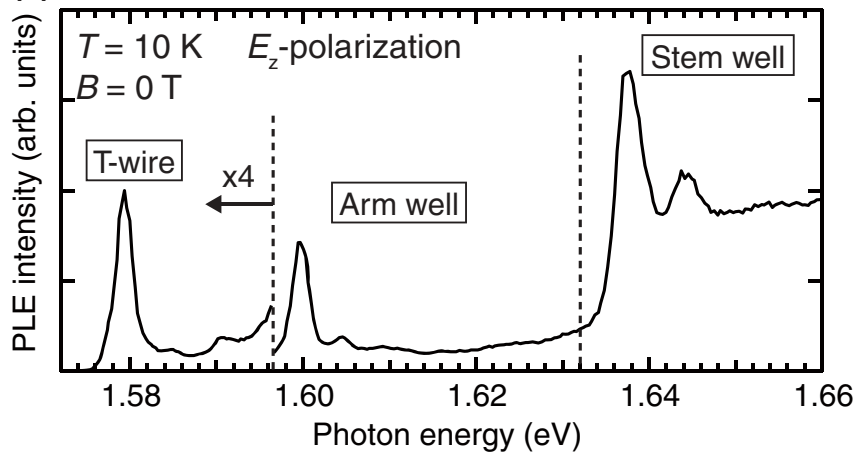

FIG. 1. (Color online) (a) Cross-sectional scanning TEM images of 100-period $T$-wire lasers. Percentages represent the $\mathrm{Al}$ content $x$ of $\mathrm{Al}_{x} \mathrm{Ga}_{1-x}$ As. $T$ wires are formed at the $T$ intersections of the 100-period stem wells and the arm well as shown in the enlarged view (upper portion). (b) Schematic of the PLE experiment configuration. (c) Typical PLE spectrum of $100 T$ wires at $0 \mathrm{~T}$ under $E_{z}$ polarization.

$100 T$-shaped intersections of 100 period (001) $\mathrm{Al}_{0.07} \mathrm{Ga}_{0.93} \mathrm{As}$ multiple quantum wells (stem wells) and a (110) GaAs quantum well (arm well). The thicknesses of the stem and arm wells were 14 and $6 \mathrm{~nm}$, respectively. In the first MBE growth on a (001) undoped GaAs substrate, we successively formed a $0.5-\mu \mathrm{m} \mathrm{GaAs}$ buffer layer, a $1-\mu \mathrm{m} \mathrm{Al}{ }_{0.5} \mathrm{Ga}_{0.5} \mathrm{As}$ sidecladding layer, a 100-period $\mathrm{Al}_{0.07} \mathrm{Ga}_{0.93} \mathrm{As} / \mathrm{Al}_{0.35} \mathrm{Ga}_{0.65} \mathrm{As}$ multiple-quantum-well structure with barrier and quantum well thicknesses of 42 and $14 \mathrm{~nm}$, respectively, a $1.0-\mu \mathrm{m}$ $\mathrm{Al}_{0.5} \mathrm{Ga}_{0.5} \mathrm{As}$ side-cladding layer, and a cap layer of $3-\mu \mathrm{m}$ GaAs. On an in situ cleaved (110) surface of the wafer, we overgrew a 6-nm GaAs quantum well (undoped) followed by a $10-\mathrm{nm} \mathrm{Al}_{0.5} \mathrm{Ga}_{0.5} \mathrm{As}$ barrier, a $175-\mathrm{nm} \mathrm{Al}_{0.12} \mathrm{Ga}_{0.88} \mathrm{As}$ optical arm, which acts as an optical core layer, a $0.96-\mu \mathrm{m}$ $\mathrm{Al}_{0.5} \mathrm{Ga}_{0.5} \mathrm{As}$ upper cladding layer, and a $10-\mathrm{nm}$ GaAs cap layer; all layers were undoped.

In the PLE measurements, the excitation light from a continuous-wave titanium-sapphire laser was focused to a spot a few micrometers in diameter on the (110) surface of the sample by using a 0.5 numerical aperture objective lens. The sample was mounted onto the cold finger in a He-flow-type cryostat. The polarization of laser light was set perpendicular $\left(E_{y}\right)$ or parallel $\left(E_{z}\right)$ to the $T$ wires for revealing the selection rule of the optical transitions. Photoluminescence (PL) was collected via the same objective lens. A polarizer positioned in a direction normal to the incident polarization reduced scattering from the excitation laser. Polarization-selective PL detections do not alter measured PLE spectral shapes, because PL occurs only from the ground-state excitons in the $T$ wires with a fixed polarization anistropy. To eliminate stray light from intense laser backscattering, we tilted the sample approximately $20^{\circ}$ off normal incidence to the backscattering geometry. The notations for the excitation-light polarizations and applied magnetic-field conditions in this work are shown in Fig. 1(b).

\section{RESULTS AND DISCUSSION}

Figure 1(c) shows a typical PLE spectrum of $T$ wires at $10 \mathrm{~K}$ in the absence of a magnetic field. The lowest PLE peak at $1.579 \mathrm{eV}$ is assigned to the first Rydberg state of the ground exciton in the $T$ wires, consisting of the first electron and first hole subbands (denoted as E1HInI). This spectrum has four other PLE peaks at 1.600, 1.604, 1.637, and $1.644 \mathrm{eV}$. These are also assigned to the heavy-hole excitons in the arm well on the core layer, in the arm well on the cladding layer, in the stem wells, and the light-hole exciton in the stem wells, respectively. ${ }^{22,30}$ The full width at half maximum of the ElHInl exciton PLE peak is about $2 \mathrm{meV}$, indicating the high uniformity of the $100 T$ wires. The continuous PLE band above $1.590 \mathrm{eV}$ comes from 1D-exciton transitions due to higher hole subbands, higher Rydberg states, and ionized continuum states. These states cannot be distinguished from each other in the absence of a magnetic field.

Figure 2 shows the magnetic-field dependence of the PLE spectra of the $T$ wires at $10 \mathrm{~K}$. These spectra were normalized at the ElHInl exciton peak. Magnetic fields were applied along the $x$ ( $B_{x}$ field, red curves) or $y$ axes $\left(B_{y}\right.$ field, blue

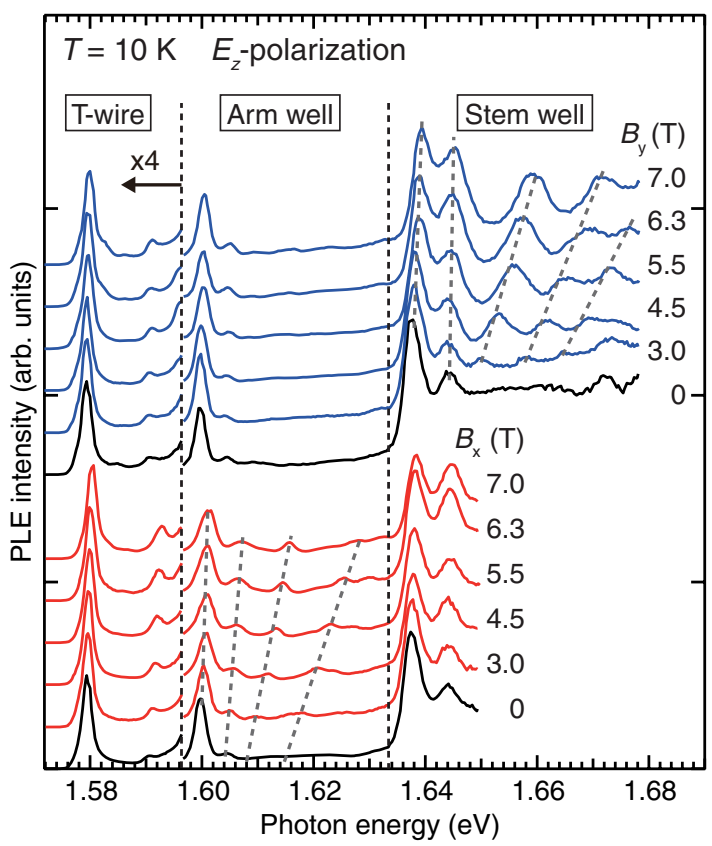

FIG. 2. (Color online) Magnetic-field dependence of PLE spectra of $100 T$ wires at $10 \mathrm{~K}$ under $E_{\mathrm{z}}$ polarization. The black curves represent the PLE spectra at $0 \mathrm{~T}$. The red and blue curves correspond to the $B_{x}$ and $B_{y}$ fields, respectively. These spectra are normalized to the lowest exciton peak. 


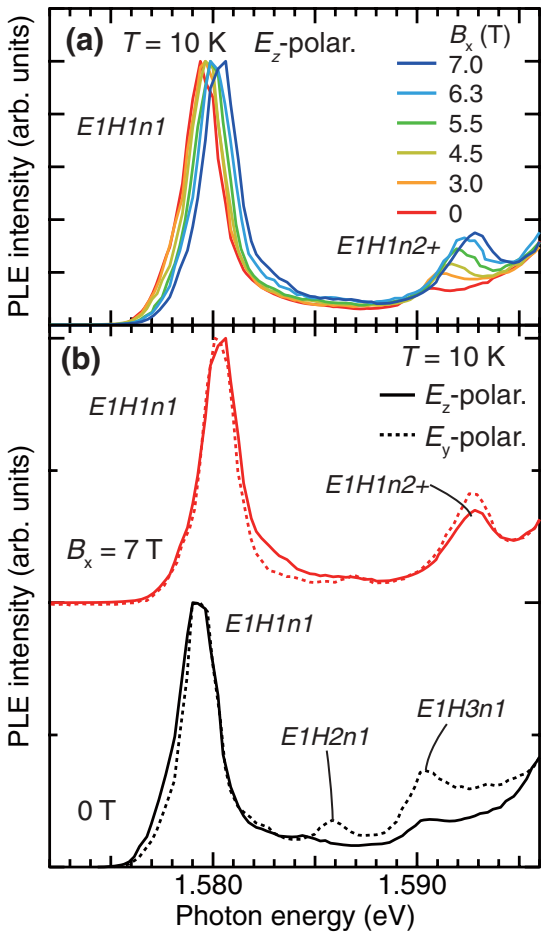

FIG. 3. (Color online) (a) $B_{x}$ dependence of PLE spectra of excitonic structures in $T$ wires at $10 \mathrm{~K}$. Excitation light is polarized to the $E_{z}$ direction. (b) Polarization dependence of the excitonic structures in $T$ wires at 0 (black curves) and $7 \mathrm{~T}$ (red curves). The $E_{z}$ and $E_{y}$ polarizations are represented by the solid and broken curves, respectively. All spectra in these figures are normalized to the lowest exciton peak.

curves), and the excitation-light polarization was parallel to the $z$ axis along the $T$ wires ( $E_{z}$ polarization). The PLE structures of the stem wells (arm well) appear to be insensitive to the $B_{x}\left(B_{y}\right)$ field, but the peaks appear due to the Landau levels and their energies blue-shift with an increase in the $B_{y}$ $\left(B_{x}\right)$ field. No significant magnetic-field dependence of the E1HInl exciton peak was observed, demonstrating that our magneto-PLE spectra provide direct experimental evidence of 1D quantum confinements, in contrast to magneto-PL spectra. ${ }^{31}$ In the PLE spectra, the unique Landau fan diagrams, dependent on the magnetic-field orientations, provide the direct assignment of the peaks to the two quantum wells and $T$ wire with different quantum-confinement configurations.

Figures 3(a) and 3(b) show enlarged views of the magneticfield dependence and the polarization dependence of the 1D exciton in the PLE spectra. The spectra were normalized at the ElHInl exciton peak around $1.580 \mathrm{eV}$. We can clearly observe that the new peak appears at the continuous edge in Fig. 3(a). The new and the ElHInl exciton peaks shift to higher energy with increasing magnetic field. The solid and broken curves correspond to the PLE spectra for $E_{z}$ and $E_{y}$ polarizations in Fig. 3(b). At $0 \mathrm{~T}$, the PLE spectra (black curves) show strong polarization dependence in the energy region between 1.585 and $1.595 \mathrm{eV}$, which includes the small peaks at 1.586 and $1.590 \mathrm{eV}$. Note that for $B_{x}=7 \mathrm{~T}$ (red curves), the polarization dependence is almost diminished.

Because it is well known that the polarization dependence of the absorption and PLE spectra in GaAs quantum wires originates from the anisotropy of hole states, ${ }^{19,20,22,32,33}$ the observed polarization-dependent peaks at 1.586 and $1.590 \mathrm{eV}$ are due to the higher hole subbands. By a previous detailed study in comparison with theoretical calculations, ${ }^{22}$ the peaks at 1.586 and $1.590 \mathrm{eV}$ at $0 \mathrm{~T}$ are assigned to the $1 \mathrm{D}$ exciton states formed with the first-subband electron and the secondor third-subband hole (denoted as ElH2nl and ElH3nI), respectively. Indeed, these contributions are stronger for $E_{y}$ polarizations, which is a polarization for the weakest $E 1 H I$ transition. In contrast, the quenched polarization dependence at $B_{x}=7 \mathrm{~T}$ indicates that the transitions due to the higher hole subbands, such as $E 1 H 2 n I$ and $E 1 H 3 n l$, are quenched. This suggests that the rigid optical transition selection rules are realized under strong magnetic fields, therefore, all absorption structures at $7 \mathrm{~T}$ are not due to the higher hole subbands but due to the ElHI transition, with ElHInl being the most common. Thus, the polarization-independent absorption peak found at $1.593 \mathrm{eV}$ under $B_{x}=7 \mathrm{~T}$ is assigned to the next optically allowed $n=2$ even-parity Rydberg state of the $E 1 H I$ excitons (denoted as $E 1 H 1 n 2+$ ) because the $n=2$ odd-parity Rydberg state of the ElHl excitons is optically forbidden. ${ }^{22}$ The energy position of second even-parity Rydberg state is in good agreement with theoretical calculation. ${ }^{22}$ The second Rydberg state has never been observed experimentally thus far.

Figure 4 shows the PLE intensity ratios of the E1HIn2+ peak (filled circles) in $E_{z}$ polarization and the $E 1 H 2 n l$ peak (open circles) in $E_{y}$ polarization to the ElHInl peak as a function of $B_{x}^{2}$. As the applied field increases, the ratio of $E 1 H 2 n 1$ to $E 1 H I n I$ excitons decreases. This indicates that the optical transition selection rules become stricter. In contrast, the ratio of ElHIn2+ to ElHInI excitons becomes larger with increasing magnetic field. By a simple model calculation for $1 \mathrm{D}$ systems, ${ }^{34}$ the magnetic-field-dependent change in the oscillator strength ratio of the second to the first Rydberg state is due to the change in the ratio of the cyclotron energy to the subband energy splitting or the lateral confinement energy. For our study samples, the lateral confinement energy corresponds

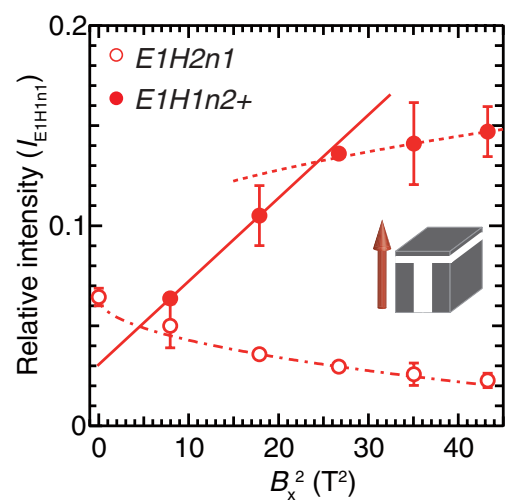

FIG. 4. (Color online) Intensity ratios of the ElH2nl (open circles) and $E 1 H 1 n 2+$ (filled circles) excitons to the EIHInl exciton as a function of the square of the magnetic field $B_{x}$. The polarizations of the laser light are $E_{z}$ for the E1HIn2+ exciton and $E_{y}$ for the $E 1 H 2 n 1$ exciton, respectively. The solid and broken lines represent the linear and the square root functions. The dash-dotted line is solely for reference. 
to the energy difference between the ground exciton in the $T$ wire and that in the arm well. When the cyclotron energy is far smaller than the zero-field subband energy splitting, the oscillator strength ratio shows quadratic dependence with $B$. However, when the cyclotron energy is far larger than the zero-field subband energy splitting, the oscillator strength ratio shows linear dependence with $B$. A crossover between the quadratic and linear regimes in the dependence on $B$ field should occur when these energies are comparable.

The experimental result can be fitted by a linear function (dotted line) at $B_{x}^{2}<30$ and a square-root function (solid line) at $B_{x}^{2}>30$, as expected by the model calculation. ${ }^{34}$ The results suggest that the cyclotron energy becomes comparable to the subband energy splitting at $5.5 \mathrm{~T}$. The cyclotron energy is estimated to be $12 \mathrm{meV}$ at $5.5 \mathrm{~T}$ using the following parameters: the reduced mass of electrons and holes $\mu=$ $0.042 m_{0}$, which is obtained by $m_{\mathrm{e}}=0.067 m_{0}$ and $m_{\mathrm{h}}=$ $0.11 m_{0} .{ }^{35}$ This energy is almost half the lateral confinement energy of $21 \mathrm{meV}$. Therefore, the observed crossover in the magnetic-field dependence appears to be reasonable. ${ }^{34}$

By interpolating the linear function to $0 \mathrm{~T}$, the oscillatorstrength ratio of the E1H1n2+ to ElHInI excitons at $0 \mathrm{~T}$ is estimated to be about $3 \times 10^{-2}$. This ratio is notably smaller than the ratio $7 \times 10^{-2}$ of the second $(2 s)$ to the first $(1 s)$ allowed Rydberg states in a two-dimensional (2D) quantum well with $6-\mathrm{nm}$ thickness. ${ }^{36}$ This comparison between the optically-allowed second-lowest Rydberg states of 1D and 2D excitons demonstrates the first quantitative observation of small oscillator strength of the second Rydberg state of 1D excitons and reflects the nature of the $1 \mathrm{D}$ exciton in which the oscillator strength is concentrated in the lowest exciton state..$^{3,4}$ It should be noted that the difference in oscillator strengths among different Rydberg states stems from the difference in the value of the squared wave function for electron-hole relative motion $r_{\mathrm{e}-\mathrm{h}}$ at the origin $r_{\mathrm{e}-\mathrm{h}}=0$. Therefore, the present small oscillator-strength ratio can be interpreted as demonstrating a remarkable contrast between the strongly and weakly bound wave functions of the ground and the high Rydberg states of $1 \mathrm{D}$ excitons.

The peak energies of the ElHInl and ElHIn2+ excitons are plotted as a function of $B^{2}$ in Fig. 5(a). The red, blue, and green circles are the data under $B_{x}, B_{y}$, and $B_{z}$ fields, respectively. The data for the E1HIn2+ exciton at $0 \mathrm{~T}$ are excluded from the graph. It is observed that the energy shifts of the $E 1 H \ln 1$ and $E 1 H 1 n 2+$ excitons are proportional to $B^{2}$ in all magnetic-field directions. These excitons show the anisotropic magnetic-field dependence due to $T$-shaped quantum confinement. By interpolating the energies of the $E 1 H \ln 1$ and $E 1 H 1 n 2+$ excitons to $0 \mathrm{~T}$, the energy difference between the two states is evaluated as $11.4 \mathrm{meV}$. This result is close to the calculated binding energy of the ground exciton, $13.5 \pm 0.5 \mathrm{meV},{ }^{37}$ indicating the weak binding of the $E 1 H 1 \mathrm{n} 2+$ exciton.

When the cyclotron radius is larger than the exciton Bohr radius, the magnetic-field dependence of the energy shift of excitons $\Delta E$, the so-called diamagnetic shift, is expressed as $\Delta E=e^{2}\left\langle r^{2}\right\rangle / 8 \mu B^{2} \equiv \beta B^{2}{ }^{38}$ Here $e$ is the elementary charge, $\left\langle r^{2}\right\rangle$ is the effective area of the excitons in the plane perpendicular to the magnetic field at $0 \mathrm{~T}$, and $\beta$ is the diamagnetic coefficient, which is a positive value. Figure 5(b)
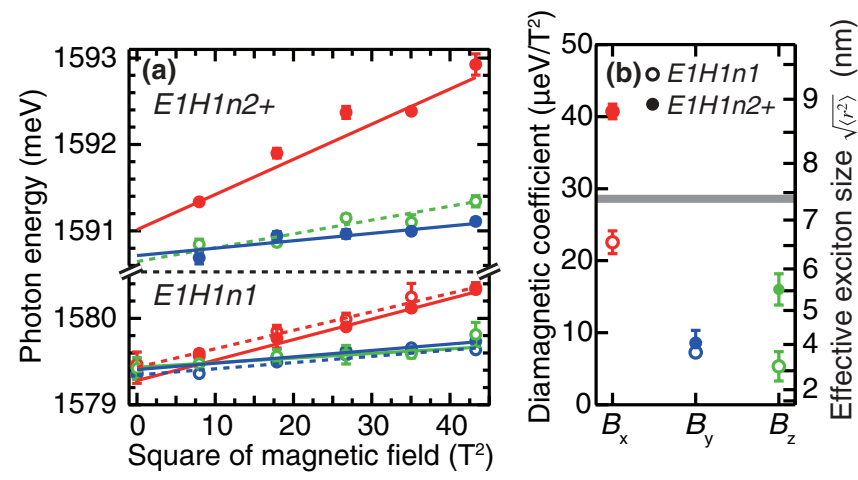

FIG. 5. (Color online) The square of the applied magnetic-field dependencies of peak positions of E1HInI and E1H1n2+ excitons. The filled (open) circles and solid (broken) lines correspond to $E_{z}$ $\left(E_{y}\right)$ polarization. (b) The diamagnetic coefficient of ElHInl (open circles) and $E 1 H \ln 2+$ (filled circles) excitons under $B_{x}, B_{y}$, and $B_{z}$ fields. The right ordinate shows the effective exciton size. Red, blue, and green circles and lines correspond to the influence of $B_{x}, B_{y}$, and $B_{z}$ fields, respectively.

plots the coefficient $\beta$, derived from Fig. 5(a), of each exciton peak as a function of magnetic-field direction. The right ordinate shows the effective exciton size, $\sqrt{\left\langle r^{2}\right\rangle}$ derived from the diamagnetic coefficients. The coefficient of the ElHInI exciton is $22.2 \mu \mathrm{eV} / \mathrm{T}^{2}$ for the $B_{x}$ field, which is smaller than the $2 \mathrm{D}$ quantum limit $\left(28.3 \mu \mathrm{eV} / \mathrm{T}^{2}\right)$, indicated by the gray hatched line. The coefficient of the ElHIn2+ exciton is $41 \mu \mathrm{eV} / \mathrm{T}^{2}$ for the $B_{x}$ field, which is larger than the $2 \mathrm{D}$ quantum limit. The coefficient of the ElHIn2+ exciton is larger than that of the ElHInl exciton in all magnetic-field directions. In particular, the coefficients of the E1HIn2+ exciton are twice as large as those of the ElHInl exciton in $B_{x}$ and $B_{z}$ fields. In addition to the polarization dependence of PLE spectra, these features strongly support the assignment that the ElH1n2+ exciton is a higher Rydberg state of the ElHInl exciton. Note, $\sqrt{\left\langle r^{2}\right\rangle}$ is determined by lateral confinement due to the heterostructure and the exciton's electron-hole Coulomb interaction. The observed difference in $\sqrt{\left\langle r^{2}\right\rangle}$ between the E1HInl and ElHIn2+ excitons is due to the difference in the exciton confinement. This also provides evidence of the contrast in binding between the ground and the higher Rydberg states of $1 \mathrm{D}$ excitons.

\section{CONCLUSION}

In summary, we determined the oscillator strength and diamagnetic shift of 1D excitons in $T$-shaped GaAs quantum wires. The oscillator strength of the E1H1n2+ exciton is $3 \times 10^{-2}$ lower than that of the ElHInl exciton. The diamagnetic coefficient of the EIHIn2+ exciton is notably larger than that of the ElHInl exciton. These results demonstrate the pronounced contrast between the ground and the higher Rydberg states as a unique and inherent characteristic of 1D excitons. Therein, only the ground exciton is strongly bound, while the higher Rydberg excitons are weakly bound. 


\section{ACKNOWLEDGMENT}

Part of this work was supported by the MEXT KAKENHI (Grants No. 20104004 and No. 20104006) and The Sumitomo Electric Industries Group CSR Foundation. This work at Princeton was partially funded by the Gordon and Betty Moore Foundation as well as the NSF MRSEC Program through the Princeton Center for Complex Materials (DMR-0819860). *okano.makoto.4a@kyoto-u.ac.jp

${ }^{1}$ H. Haug and S. W. Koch, Quantum Theory of the Optical and Electronic Properties of Semiconductors, 5th ed. (World Scientific, Singapore, 2009).

${ }^{2}$ R. Loudon, Am. J. Phys. 27, 649 (1959).

${ }^{3}$ T. Ogawa and T. Takagahara, Phys. Rev. B 44, 8138 (1991).

${ }^{4}$ T. Ando, J. Phys. Soc. Jpn. 74, 777 (2005), and references therein.

${ }^{5}$ E. Kapon, D. M. Hwang, and R. Bhat, Phys. Rev. Lett. 63, 430 (1989).

${ }^{6}$ M. Yoshita, S. M. Liu, M. Okano, Y. Hayamizu, H. Akiyama, L. N. Pfeiffer, and K. W. West, J. Phys.: Condens. Matter 19, 295217 (2007), and references therein.

${ }^{7}$ F. Wang, G. Dukovic, L. E. Brus, and T. F. Heinz, Science 308, 838 (2005); J. Maultzsch, R. Pomraenke, S. Reich, E. Chang, D. Prezzi, A. Ruini, E. Molinari, M. S. Strano, C. Thomsen, and C. Lienau, Phys. Rev. B 72, 241402 (2005).

${ }^{8}$ J. Lefebvre and P. Finnie, Nano Lett. 8, 1890 (2008).

${ }^{9}$ Y. Kanemitsu, Phys. Chem. Chem. Phys. 13, 14879 (2011), and references therein

${ }^{10}$ T. Hasegawa, Y. Iwasa, H. Sunamura, T. Koda, Y. Tokura, H. Tachibana, M. Matsumoto, and S. Abe, Phys. Rev. Lett. 69, 668 (1992).

${ }^{11}$ L. N. Pfeiffer, K. W. West, H. L. Stormer, J. P. Eisenstein, K. W. Baldwin, D. Gershoni, and J. Specter, Appl. Phys. Lett. 56, 1697 (1990).

${ }^{12}$ M. Yoshita, H. Akiyama, L. N. Pfeiffer, and K. W. West, Appl. Phys. Lett. 81, 49 (2002).

${ }^{13}$ M. J. O'Connell, S. M. Bachilo, C. B. Huffman, V. C. Moore, M. S. Strano, E. H. Haroz, K. L. Rialon, P. J. Boul, W. H. Noon, C. Kittrell, J. Ma, R. H. Hauge, R. B. Weisman, and R. E. Smalley, Science 297, 593 (2002).

${ }^{14}$ X. Tu, S. Manohar, A. Jagota, and M. Zheng, Nature 460, 250 (2009).

${ }^{15}$ For example, L. A. Coldren, S. W. Corzine, and M. L. Mashanovitch, Diode Lasers and Photonic Integrated Circuits, 2nd ed. (Wiley, New York, 2012).

${ }^{16}$ R. Rinaldi, R. Cingolani, M. Lepore, M. Ferrara, I. M. Catalano, F. Rossi, L. Rota, E. Molinari, P. Lugli, U. Marti, D. Martin, F. Morier-Gemoud, P. Ruterana, and F. K. Reinhart, Phys. Rev. Lett. 73, 2899 (1994).

${ }^{17}$ H. Weman, M. Potemski, M. E. Lazzouni, M. S. Miller, and J. L. Merz, Phys. Rev. B 53, 6959 (1996).

${ }^{18}$ W. R. Tribe, M. J. Steer, D. J. Mowbray, M. S. Skolnick, A. N. Forshaw, J. S. Roberts, G. Hill, M. A. Pate, C. R. Whitehouse, and G. M. Williams, App. Phys. Lett. 70, 993 (1997).
${ }^{19}$ T. Sogawa, H. Ando, S. Ando, and H. Kanbe, Phys. Rev. B 56, 1958 (1997).

${ }^{20}$ F. Vouilloz, D. Y. Oberli, M.-A. Dupertuis, A. Gustafsson, F. Reinhardt, and E. Kapon, Phys. Rev. Lett. 78, 1580 (1997).

${ }^{21}$ M. Bayer, Ch. Schlier, Ch. Gréus, A. Forchel, S. Benner, and H. Haug, Phys. Rev. B 55, 13180 (1997).

${ }^{22}$ H. Itoh, Y. Hayamizu, M. Yoshita, H. Akiyama, L. N. Pfeiffer, K. W. West, M. H. Szymanska, and P. B. Littlewood, App. Phys. Lett. 83, 2043 (2003).

${ }^{23}$ N. I. Cade, R. Roshan, M. Hauert, A. C. Maciel, J. F. Ryan, A. Schwarz, Th. Schäpers, and H. Lüth, Phys. Rev. B 70, 195308 (2004).

${ }^{24}$ S. Palmgren, H. Weman, A. Schoenberg, K. F. Karlsson, M.-A. Dupertuis, K. Leifer, A. Rudra, and E. Kapon, Appl. Phys. Lett. 89, 191111 (2006)

${ }^{25}$ F. Wang, G. Dukovic, L. E. Brus, and T. F. Heinz, Phys. Rev. Lett. 92, 177401 (2004).

${ }^{26}$ V. I. Klimov, A. A. Mikhailovsky, D. W. McBranch, C. A. Leatherdale, and M. G. Bawendi, Science 287, 1011 (2000).

${ }^{27}$ Y.-Z. Ma, L. Valkunas, S. L. Dexheimer, S. M. Bachilo, and G. R. Fleming, Phys. Rev. Lett. 94, 157402 (2005): L. Huang and T. D. Krauss, Phys. Rev. Lett. 96, 057407 (2006).

${ }^{28}$ A. Ueda, K. Matsuda, T. Tayagaki, and Y. Kanemitsu, App. Phys. Lett. 92, 233105 (2008); S. Wang, M. Khafizov, X. Tu, M. Zheng, and T. D. Krauss, Nano Lett. 10, 2381 (2010).

${ }^{29}$ N. M. Gabor, Z. Zhong, K. Bosnick, J. Park, and P. L. McEuen, Science 325, 1367 (2009).

${ }^{30}$ H. Akiyama, M. Yoshita, L. N. Pfeiffer, K. W. West, and A. Pinczuk, App. Phys. Lett. 82, 379 (2003).

${ }^{31}$ T. Someya, H. Akiyama, and H. Sakaki, Phys. Rev. Lett. 74, 3664 (1995); Solid State Commun. 108, 923 (1998).

${ }^{32}$ M. A. Dupertuis, E. Martinet, D. Y. Oberli, and E. Kapon, Europhys. Lett. 52, 420 (2000).

${ }^{33}$ S. Watanabe, M. Yoshita, S. Koshiba, and H. Akiyama, Jpn. J. Appl. Phys. 41, 5924 (2002).

${ }^{34}$ S. Benner and H. Haug, Phys. Rev. B 47, 15750 (1993).

${ }^{35}$ M. Okano, P. Huai, M. Yoshita, S. Inada, H. Akiyama, K. Kamide, K. Asano, and T. Ogawa, J. Phys. Soc. Jpn. 80, 114716 (2011).

${ }^{36}$ M. Gurioli, J. Martinez-Pastor, M. Colocci, A. Bosacchi, S. Franchi, and L. C. Andreani, Phys. Rev. B 47, 15755 (1993).

${ }^{37}$ M. H. Szymanska, P. B. Littlewood, and R. J. Needs, Phys. Rev. B 63, 205317 (2001).

${ }^{38}$ O. Akimoto and H. Hasegawa, J. Phys. Soc. Jpn. 22, 181 (1967). 